\title{
Correction: Whole exome sequencing study identifies novel rare and common Alzheimer's-Associated variants involved in immune response and transcriptional regulation
}

Joshua C. Bis ${ }^{1} \cdot$ Xueqiu Jian ${ }^{2} \cdot$ Brian W. Kunkle ${ }^{3} \cdot$ Yuning Chen $\mathbb{1}^{4} \cdot$ Kara L. Hamilton-Nelson ${ }^{3} \cdot$ William S. Bush ${ }^{5}$. William J. Salerno ${ }^{6}$ - Daniel Lancour (10) ${ }^{7}$ Yiyi $\mathrm{Ma}^{7} \cdot$ Alan E. Renton $\mathbb{1 0}^{8} \cdot$ Edoardo Marcora $^{8,9} \cdot$ John J. Farrell $^{7}$. Yi Zhao ${ }^{10}$ - Liming Qu ${ }^{10}$. Shahzad Ahmad ${ }^{11}$ - Najaf Amin ${ }^{12}$. Philippe Amouyel $\mathbb{C}^{12,13,14} \cdot$ Gary W. Beecham ${ }^{3}$. Jennifer E. Below ${ }^{15}$ - Dominique Campion ${ }^{16,17}$. Laura Cantwell ${ }^{10}$. Camille Charbonnier ${ }^{16}$. Jaeyoon Chung $\mathbb{D}^{7}$. Paul K. Crane ${ }^{1} \cdot$ Carlos Cruchaga $^{18} \cdot$ L. Adrienne Cupples ${ }^{4,19} \cdot$ Jean-François Dartigues $^{20} \cdot$ Stéphanie Debette $^{20,21}$. Jean-François Deleuze ${ }^{22}$ - Lucinda Fulton ${ }^{23}$ - Stacey B. Gabriel ${ }^{24}$ - Emmanuelle Genin ${ }^{25}$ - Richard A. Gibbs ${ }^{6}$. Alison Goate $\mathbb{1}^{8,9} \cdot$ Benjamin Grenier-Boley $^{12} \cdot$ Namrata Gupta $^{24} \cdot$ Jonathan L. Haines $^{5} \cdot$ Aki S. Havulinna ${ }^{26,27}$. Seppo Helisalmi ${ }^{28}$ - Mikko Hiltunen ${ }^{29}$. Daniel P. Howrigan ${ }^{30,31}$ - M. Arfan Ikram $\mathbb{1}^{11}$ - Jaakko Kaprio $\mathbb{1}^{26}$. Jan Konrad ${ }^{18} \cdot$ Amanda Kuzma $^{10}$ - Eric S. Lander ${ }^{24} \cdot$ Mark Lathrop $^{32} \cdot$ Terho Lehtimäki $^{33} \cdot$ Honghuang Lin $^{34}$. Kari Mattila ${ }^{33} \cdot$ Richard Mayeux $^{35} \cdot$ Donna M. Muzny $^{6} \cdot$ Waleed Nasser $^{6} \cdot$ Benjamin Neale $^{30,31} \cdot$ Kwangsik Nho $^{36}$. Gaël Nicolas $\mathbb{1}^{16} \cdot$ Devanshi Patel $^{7} \cdot$ Margaret A. Pericak-Vance ${ }^{3} \cdot$ Markus Perola ${ }^{26,27,37} \cdot$ Bruce M. Psaty ${ }^{1,38,39,40}$. Olivier Quenez ${ }^{16} \cdot$ Farid Rajabli $^{3} \cdot$ Richard Redon $^{41} \cdot$ Christiane Reitz $^{35} \cdot$ Anne M. Remes $^{28,42} \cdot$ Veikko Salomaa $^{27}$. Chloe Sarnowski $^{4} \cdot$ Helena Schmidt ${ }^{43} \cdot$ Michael Schmidt $^{3} \cdot$ Reinhold Schmidt $^{43} \cdot$ Hilkka Soininen $^{28,44}$.

Timothy A. Thornton ${ }^{45}$. Giuseppe Tosto ${ }^{35}$. Christophe Tzourio ${ }^{20}$. Sven J. van der Lee $\mathbb{C}^{11}$. Cornelia M. van Duijn ${ }^{11}$. Otto Valladares $^{10} \cdot$ Badri Vardarajan $^{35} \cdot$ Li-San Wang $^{10} \cdot$ Weixin Wang $^{10} \cdot$ Ellen Wijsman ${ }^{46,47} \cdot$ Richard K. Wilson $^{23}$. Daniela Witten ${ }^{45,47} \cdot$ Kim C. Worley ${ }^{6}$ - Xiaoling Zhang ${ }^{4,7}$ - Alzheimer's Disease Sequencing Project •

Celine Bellenguez $\mathbb{D}^{12}$ - Jean-Charles Lambert $\mathbb{D}^{12} \cdot$ Mitja I. Kurki ${ }^{26,30,31}$ - Aarno Palotie $e^{26,30,31} \cdot$ Mark Daly $\mathbb{D}^{24,26,31}$. Eric Boerwinkle ${ }^{6,48} \cdot$ Kathryn L. Lunetta $\mathbb{1}^{4} \cdot$ Anita L. Destefano $0^{4,49} \cdot$ Josée Dupuis $^{4}$ - Eden R. Martin ${ }^{3}$.

Gerard D. Schellenberg $^{10} \cdot$ Sudha Seshadri $^{19,49,50} \cdot$ Adam C. Naj $\mathbb{1}^{10} \cdot$ Myriam Fornage $^{2,48} \cdot$ Lindsay A. Farrer $^{4,7,49,51,52}$

Published online: 21 October 2019

(c) The Author(s) 2019. This article is published with open access

\section{Correction to: Molecular Psychiatry} https://doi.org/10.1038/s41380-018-0112-7 published online 14 August 2018

Following publication, the authors noticed that 'Laura Cantwell', 'Otto Valladares', and 'Li-San Wang' were inadvertently omitted from the author list. These authors have now been added to the author list in 21 st, 77 th, and 79th position, respectively. This has been corrected in both the PDF and HTML versions of the article.

These authors contributed equally: Joshua C. Bis, Xueqiu Jian, Brian W. Kunkle, Yuning Chen

These authors equally supervised the study: Adam C. Naj, Myriam Fornage, Lindsay A. Farrer

Lindsay A. Farrer

farrer@bu.Edu

Extended author information available on the last page of the article 
1 Department of Medicine (General Internal Medicine), University of Washington, Seattle, WA, USA

2 Institute of Molecular Medicine, McGovern Medical School, University of Texas Health Science Center at Houston, Houston, TX, USA

3 John P. Hussman Institute for Human Genomics, Miller School of Medicine, University of Miami, Miami, FL, USA

4 Departments of Biostatistics, Boston University School of Public Health, Boston, MA, USA

5 Case Western Reserve University, Cleveland Heights, OH, USA

6 Human Genome Sequencing Center and Department of Molecular and Human Genetics, Baylor College of Medicine, Houston, TX, USA

7 Department of Medicine (Biomedical Genetics), Boston University School of Medicine, Boston, MA, USA

8 Department of Neuroscience and Ronald M Loeb Center for Alzheimer's Disease, Icahn School of Medicine at Mount Sinai, New York, NY, USA

9 Department of Genetics and Genomics Sciences, Icahn School of Medicine at Mount Sinai, New York, NY, USA

10 University of Pennsylvania Perelman School of Medicine, Philadelphia, PA, USA

11 Erasmus University Medical Center, Rotterdam, Netherlands

12 Inserm, U1167, RID-AGE-Risk Factors and Molecular Determinants of Aging-Related Diseases, Lille, France

13 Institut Pasteur de Lille, Lille, France

14 University Lille, U1167-Excellence Laboratory LabEx DISTALZ, Lille, France

15 Department of Medical Genetics, Vanderbilt University Medical Center, Nashville, TN, USA

16 Department of Genetics and CNR-MAJ, Normandie Université, UNIROUEN, Inserm U1245 and Rouen University Hospital, F 76000, Normandy Centre for Genomic and Personalized Medicine, Rouen, France

17 Department of Research, Centre Hospitalier du Rouvray, Sotteville-lès-, Rouen, France

18 Department of Psychiatry, Washington University, St. Louis, MO, USA

19 National Heart, Lung, and Blood Institute's Framingham Heart Study, Framingham, MA, USA

20 University of Bordeaux, Inserm, Bordeaux Population Health Research Center, team VINTAGE, UMR 1219, F-33000 Bordeaux, France

21 Department of Neurology and Institute for Neurodegenerative Diseases, Bordeaux University Hospital, Memory Clinic, F-33000 Bordeaux, France

22 Centre National de Recherche en Génomique Humaine, Institut François Jacob, Direction de le Recherche Fondamentale, CEA, Evry, France
23 McDonnell Genome Institute, Washington University, St. Louis, MO, USA

24 Broad Institute of MIT and Harvard, Cambridge, MA, USA

25 Inserm UMR-1078, CHRU Brest, Université Brest, Brest, France

26 Institute for Molecular Medicine Finland (FIMM), University of Helsinki, Helsinki, Finland

27 National Institute for Health and Welfare, Helsinki, Finland

28 Institute of Clinical Medicine - Neurology and Department of Neurology, University of Eastern Finland, Kuopio, Finland

29 Institute of Biomedicine, University of Eastern Finland, Kuopio, Finland

30 Program in Medical and Population Genetics and Genetic Analysis Platform, Stanley Center for Psychiatric Research, Broad Institute of MIT and Harvard, Cambridge, MA, USA

31 Psychiatric \& Neurodevelopmental Genetics Unit, Massachusetts General Hospital, Boston, MA, USA

32 McGill University and Génome Québec Innovation Centre, Montréal, Canada

33 Department of Clinical Chemistry, Fimlab Laboratories and Finnish Cardiovascular Research Center-Tampere, Faculty of Medicine and Life Sciences, University of Tampere, Tampere, Finland

34 Department of Medicine (Computational Biomedicine), Boston University School of Medicine, Boston, MA, USA

35 Columbia University, New York, NY, USA

36 Indiana University School of Medicine, Indianapolis, IN, USA

37 University of Tartu, Estonian Genome Center, Tartu, Estonia

38 Department of Epidemiology, University of Washington, Seattle, WA, USA

39 Department of Health Services, University of Washington, Seattle, WA, USA

40 Kaiser Permanente Washington Health Research Institute, Seattle, WA, USA

41 Inserm, CNRS, Univ. Nantes, CHU Nantes, l'institut du thorax, Nantes, France

42 Unit of Clinical Neuroscience, Neurology, University of Oulu and Medical Research Center, Oulu University Hospital, Oulu, Finland

43 Department of Neurology, Clinical Division of Neurogeriatrics, Medical University of Graz, Graz, Austria

44 Department of Neurology, Kuopio University Hospital, Kuopio, Finland

45 Department of Statistics, University of Washington, Seattle, WA, USA

46 Department of Medicine (Medical Genetics), University of Washington, Seattle, WA, USA

47 Department of Biostatistics, University of Washington, Seattle, WA, USA 
48 School of Public Health, University of Texas Health Science Center at Houston, Houston, TX, USA

49 Departments of Neurology, Boston University School of Medicine, Boston, MA, USA

50 Glenn Biggs Institute for Alzheimer's and Neurodegenerative Diseases, University of Texas Health Sciences Center, San Antonio, TX, USA

51 Department of Epidemiology, Boston University School of Public Health, Boston, MA, USA

52 Department of Ophthalmology, Boston University School of Medicine, Boston, MA, USA
Open Access This article is licensed under a Creative Commons Attribution 4.0 International License, which permits use, sharing, adaptation, distribution and reproduction in any medium or format, as long as you give appropriate credit to the original author(s) and the source, provide a link to the Creative Commons license, and indicate if changes were made. The images or other third party material in this article are included in the article's Creative Commons license, unless indicated otherwise in a credit line to the material. If material is not included in the article's Creative Commons license and your intended use is not permitted by statutory regulation or exceeds the permitted use, you will need to obtain permission directly from the copyright holder. To view a copy of this license, visit http://creativecommons. org/licenses/by/4.0/. 